\title{
Physics of cuprates with the two-band Hubbard model - The validity of the one-band Hubbard model
}

\author{
A. Macridin and M. Jarrell \\ University of Cincinnati, Cincinnati, Ohio, 45221, USA \\ Th. Maier \\ Computer Science and Mathematics Division, Oak Ridge National Laboratory, Oak Ridge, Tennessee 37831, USA \\ G. A. Sawatzky \\ University of British Columbia, 6224 Agricultural Road, Vancouver, BC V6T 1Z1, Canada
}

(Dated: August 27, 2018)

\begin{abstract}
We calculate the properties of the two-band Hubbard model using the Dynamical Cluster Approximation. The phase diagram resembles the generic phase diagram of the cuprates, showing a strong asymmetry with respect to electron and hole doped regimes, in agreement with experiment. Asymmetric features are also seen in one-particle spectral functions and in the charge, spin and dwave pairing susceptibility functions. We address the possible reduction of the two-band model to a low-energy single-band one, as it was suggested by Zhang and Rice. Comparing the two-band Hubbard model properties with the single-band Hubbard model ones, we have found similar low-energy physics provided that the next-nearest-neighbor hopping term $t^{\prime}$ has a significant value $\left(t^{\prime} / t \approx 0.3\right)$. The parameter $t^{\prime}$ is the main culprit for the electron-hole asymmetry. However, a significant value of $t^{\prime}$ cannot be provided in a strict Zhang and Rice picture where the extra holes added into the system bind to the existing $\mathrm{Cu}$ holes forming local singlets. We notice that by considering approximate singlet states, such as plaquette ones, reasonable values of $t^{\prime}$, which capture qualitatively the physics of the two-band model can be obtained. We conclude that a single-band t-t'-U Hubbard model captures the basic physics of the cuprates concerning superconductivity, antiferromagnetism, pseudogap and electron-hole asymmetry, but is not suitable for a quantitative analysis or to describe physical properties involving energy scales larger than about $0.5 \mathrm{eV}$.
\end{abstract}

\section{INTRODUCTION}

The theory of the cuprate high temperature superconductors remains one of the most important and daunting problems in condensed matter physics. The high $T_{c}$ cuprate superconductors are layered materials with relatively complex structures and chemical composition. They are highly correlated, with an effective bandwidth roughly equal to the effective local Coulomb interaction. The short-range correlations are known to play a paramount role in these materials. Therefore, the Dynamical Cluster Approximation (DCA $)^{\underline{1}}$, which treats short-range correlations explicitly and the long-range physics at the mean-field level, is an ideal tool for the investigation of these systems.

A common characteristic all the cuprate materials share is the presence of quasi-two-dimensional $\mathrm{CuO}_{2}$ planes. These planes are commonly believed to contain the low-energy physics. However, the full complexity of the orbital chemistry of just the $\mathrm{CuO}_{2}$ planes and the strong Coulomb repulsion on the $\mathrm{Cu}$ ions would lead to models which are very difficult to study with conventional techniques.

The cuprates are characterized by a very rich, but also in many respects very intriguing physics. The undoped materials are antiferromagnetic (AFM) insulators with a gap of approximatively $2 \mathrm{eV}$. Upon doping the AFM is destroyed and the system becomes superconducting (SC). At small doping, in the proximity of the AFM phase, the normal state physics cannot be described in terms of Fermi liquid theory and is characterized by the presence of a pseudogap. An essential demand of every successful theory is to capture all these fundamental features at the same time.

Experimental data show that the phase diagram and other physical characteristics like the density of states (DOS) near the Fermi level of the hole-doped and electron-doped materials are very different 2.3 .4 . There could be many reasons for this asymmetry. The electron and the hole doped materials are physically different and apart from the $\mathrm{CuO}$ planes, they contain different elements and chemical structures. These structural and compositional differences can influence the low-energy physics. Therefore in this paper, we use DCA to address whether the physics of a pure $\mathrm{CuO}$ plane contains this asymmetry or if the origin of the asymmetry in real materials comes from other influences.

Different models for describing the physics of a $\mathrm{CuO}$ plane were proposed by various authors. Photoemission experiments in the insulating parent material show that the first electron-removal states have primarily oxygen character; whereas, the first electron-addition states have $d$ character, already suggesting a strong asymmetry. This places these materials in the charge-transfer gap region of the Zaanen-Sawatzky-Allen scheme ${ }^{5}$. Early on, considering the ligand field symmetry and band structure calculations ${ }^{6.7 .8}$, it was realized that the most important degrees of freedom are the $\mathrm{Cu} d_{x^{2}-y^{2}}$ which couple with 
the in-plane $\mathrm{O} p$ orbitals. Therefore, one of the first models proposed to describe the physics of high $T_{c}$ materials was the so called three-band Hubbard model introduced by Varma et $a l^{\underline{9}}$ and Emery et al $l^{10}$, which considers explicitly both the oxygen $p_{\sigma}$ and the cooper $d_{x^{2}-y^{2}}$ orbitals. In fact, because the direct oxygen-oxygen hopping is neglected, only the combination of oxygen orbitals with $x^{2}-y^{2}$ symmetry couples with the $d$ orbitals, and the above proposed three-band model reduces to a two-band one.

However Zhang and Rice $(\mathrm{ZR}) \underline{11}$ argue that the lowenergy physics of the hole doped superconductors can be described by a single-band model. Starting from the twoband model, Zhang and Rice claim that an extra hole added into the oxygen band binds strongly with a hole on the $C u$, forming an on-site singlet. This singlet state, which has zero spin can be thought as moving through the lattice like a hole in an antiferromagnetic background. Consequently, the physics can be described by a one-band t-J model.

Pertinent criticism to these simplified models were raised by various authors. With respect to $\mathrm{Cu}$ degrees of freedom, H. Eskes et al $\stackrel{12}{n}$ stressed the possible importance of the other $d$ orbitals, showing that they should be explicitly considered when physics which implies excitations with energy larger than $\approx 1 \mathrm{eV}$ is involved. However, these criticisms do not concern us for the present study since we are interested only in physics at energies lower than $\approx 0.5 \mathrm{eV}$.

Investigating the relative importance of various parameters describing the $\mathrm{CuO}_{2}$ planes it was realized early on that in addition to the $\mathrm{Cu}$ on-site Coulomb repulsion $\left(U_{d d} \approx 8 \mathrm{eV}\right)$ and $\mathrm{Cu}$-O hopping integral $\left(t_{p d} \approx\right.$ $1.3-1.5 \mathrm{eV})$ the $\mathrm{O}-\mathrm{O}$ hopping integrals result in a large O $2 p$ band width $(W \approx 5 \mathrm{eV})$, indicating that these should be included explicitly in any theory 12.13.14. Therefore, using the DCA technique as a means of including all these most important parameters and bands, we address two major problems in this paper: The physics of the $\mathrm{CuO}_{2}$ plane (including a detailed study of the electronhole asymmetry) and the reduction of the multi-band model to a single-band model.

Regarding the reduction to a one-band model, one of the most serious criticisms to ZR theory is the neglect of the $\mathrm{O} 2 p$ band structure 15.16 . The natural tendency of the finite oxygen bandwidth is to delocalize and destabilize the ZR singlets. The question arises whether the low-energy states (i.e. the ZR singlets) are still well separated from the higher energy states (i.e. the non-bonding oxygen states). Otherwise, the reduction to a single-band model which neglects these high energy states is not possible. This problem was previously considered by Eskes and Sawatzky ${ }^{16}$ within an impurity calculation approach, but there, unlike in the DCA approach, both the spatial correlation effects and the dispersion of the low-energy states were neglected.

Another important objection to ZR theory was first raised by Emery and Reiter 17 , and regards the nature of the low-energy states. Are these states real singlets which can be mapped onto holes, or does the hole on the $\mathrm{O}$ bind into a more complicated state which involves more than one $\mathrm{Cu}$ hole? Choosing a particular solvable example, which considers the $\mathrm{Cu}$ spins arranged ferromagnetically, they showed that the low-energy states are in fact an admixture of the Zhang-Rice singlets and the corresponding triplets. This implies a nonzero value for the oxygen spin, and destroys the equivalence of these states to holes. However, it is not clear if the situation is similar in the cuprates, i.e. if the ZR singlet-ZR triplet admixture is significant. But the merit of Emery and Reiter is to emphasize that the fact that, as a consequence of the strong $\mathrm{Cu}-\mathrm{O}$ hybridization low-energy states well separated from the non-bonding oxygen band states appear, does not necessarily mean that the physics can be reduced to a single-band model.

The third problem we address regarding the reduction to a single-band model is the estimation of the singleband parameters. We notice that different approximations result in different values of the parameters. Especially the magnitude of the next-nearest-neighbor hopping is very dependent of the initial assumptions. For example, if we assume that the hole addition low-energy states are genuine ZR singlets, i.e bound states between a $\mathrm{Cu}$ hole and a orthogonal Wannier oxygen orbital, we obtain a negligible next-nearest-neighbor hopping $\frac{18}{}$. On the other hand, if we consider the low-energy states to be plaquette singlets, i.e. bound states between a $\mathrm{Cu}$ hole and a hole on the state formed by the four oxygen around the $\mathrm{Cu}$, the value of the next-nearest-neighbor hopping is significant ${ }^{19}$. Of course, because of the nonorthogonality of the plaquette states, the plaquette singlets are not genuine singlets and therefore they cannot be rigorously mapped into holes. However, because their overlap with the local singlets is large $(96 \%)^{11.17}$, it is still possible that this approximation is good.

Our calculations show that a multi-band model and a single-band t-t'-U Hubbard model with a significant value of the next-nearest-neighbor hopping exhibit a similar low-energy physics. The essential parameter needed for the agreement is the next-nearest-neighbor hopping, $t^{\prime}$. This parameter is also the main culprit for the observed electron-hole asymmetry. However, as mentioned above, the large value of $t^{\prime}$ cannot be obtained in a strict ZR picture. Thus our results also implicitly indicate that the multi-band model cannot be rigorously reduced to a single-band model. Therefore, besides showing the similarities between the two models, we also point out their significant differences in this paper.

The final conclusion is that a single-band t-t'-U Hubbard model, with a significant value of $t^{\prime}$, captures the basic physics of the cuprates, and thus is suitable to describe the AFM, pseudogap and SC physics together with the relevant asymmetries observed in the phase diagram, in the one-particle spectra and in the two-particle response functions. However, we believe that it is not suitable for a quantitative material specific analysis, for 
describing the higher energy spectroscopic features as in optical spectroscopy or resonant inelastic x-ray scattering, or for studying more subtle features related to the finite value of the spin on the oxygen.

This paper is organized as follows. In Section III the two-band Hubbard model and the DCA technique is introduced. Our two-band model takes fully into account the oxygen dispersion and considers only the oxygen degrees of freedom which couple directly to the $\mathrm{Cu} d_{x^{2}-y^{2}}$ orbitals. The results of the DCA calculation applied to the two-band Hamiltonian are presented in Section III] The possible reduction of the two-band model to a singleband one, together with a detailed analysis of the singleband t-t'-U Hubbard model, is addressed in Section IV] A discussion regarding the similarities and the differences between the two-band model and the single-band one is given in Section $\mathrm{V}$ The conclusions of our study are reviewed in Section VI]

\section{FORMALISM}

\section{A. The model Hamiltonian}

Band structure calculations ${ }^{14.20}$, cluster calculation ${ }^{12}$, photoemission ${ }^{12}$ and other experiments show that the relevant $\mathrm{Cu}$ degrees of freedom are the $d_{x^{2}-y^{2}}$ orbitals which couple with the in-plane $p_{x}$ and $p_{y} \mathrm{O}$ orbitals. All these degrees of freedom result in a five-band (four oxygen and one copper band) Hamiltonian in general. We have studied the five-band model in detai ${ }^{21}$ and have found that due to the strong $\mathrm{Cu}-\mathrm{O}$ hybridization, only the oxygen degrees of freedom which couple directly with $\mathrm{Cu}$ are relevant for the low-energy physics. Consequently, to a very good approximation, the five-band model can be reduced to a two-band one.

The two-band model contains one $\mathrm{Cu} d_{x^{2}-y^{2}}$ correlated band and one oxygen band. At every site the oxygen states are obtained by taking a linear combination with $x^{2}-y^{2}$ symmetry of the four $\mathrm{O} p_{\sigma}$ orbitals which form a plaquette around the $\mathrm{Cu}$ ion. These are the only oxygen states which hybridize directly with $\mathrm{Cu}$. However, it should be mentioned that these plaquette states are not orthogonal, two neighboring states sharing a common oxygen atom. An orthogonal basis can be obtained by the procedure described in the original ZR paper ${ }^{11}$. First, applying a Fourier transform, translational invariant (Bloch) states are constructed. The Bloch states are orthogonal but not normalized, so they should be multiplied by a normalization factor

$$
\beta(k)=\left[\sin ^{2}\left(k_{x} / 2\right)+\sin ^{2}\left(k_{y} / 2\right]^{-1 / 2} .\right.
$$

After normalization a complete and orthonormal set of oxygen states is obtained.

In this basis the two-band Hubbard Hamiltonian can be written as:

$H=\sum_{k, \sigma} E(k) c_{k \sigma}^{\dagger} c_{k \sigma}+E_{d} d_{k \sigma}^{\dagger} d_{k \sigma}+V(k)\left(c_{k \sigma}^{\dagger} d_{k \sigma}+h . c\right)$

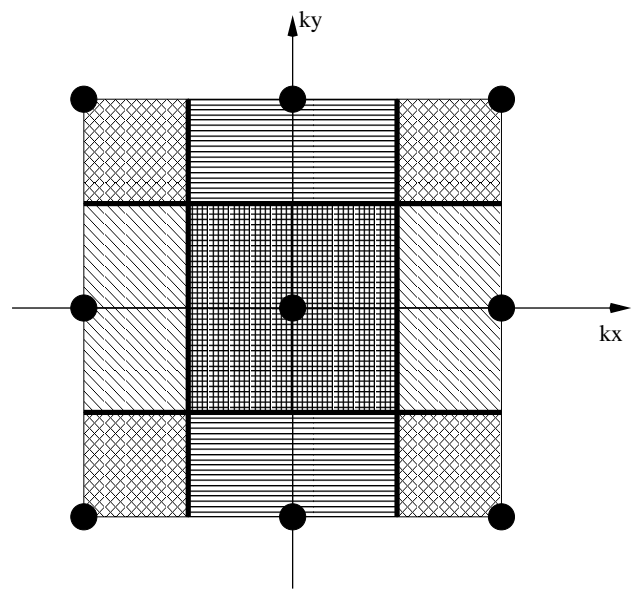

FIG. 1: Coarse-graining of the Brillouin Zone in four cells $\left(N_{c}=4\right)$ around $K=(0,0),(0, \pi),(\pi, 0)$ and $(\pi, \pi)$.

$$
+U \sum_{i} n_{d i \uparrow} n_{d i \downarrow}
$$

We work in the hole representation and $d_{k \sigma}^{\dagger}\left(c_{k \sigma}^{\dagger}\right)$ represents the creation operator of a $\mathrm{Cu}(\mathrm{O})$ hole with spin $\sigma$ and momentum $k$. The $\mathrm{O}$ band dispersion and the $\mathrm{Cu}-\mathrm{O}$ hybridization are given by

$$
\begin{gathered}
E(k)=E_{p}-8 t_{p p} \beta^{2}(k) \sin ^{2}\left(k_{x} / 2\right) \sin ^{2}\left(k_{y} / 2\right) \\
V(k)=2 t_{p d} \beta^{-1}(k)
\end{gathered}
$$

with $t_{p p}$ being the O-O hopping integral. The last term in Eq2 2 represents the Coulomb repulsion between two holes on the same $d$ orbital. We choose the commonly accepted values of the parameters, based on the band structure calculations of McMahan et al. ${ }^{20}$ and Hybertsen et al ${ }^{14}$ Because of the low density of oxygen holes $(25 \%-30 \%)$, we treat the Coulomb repulsion on $O$ (given by $U_{p p}$ ) and the repulsion between nearest-neighbor $\mathrm{Cu}$ and $\mathrm{O}$ holes (given by $U_{p d}$ ) at the mean field level as a reasonable approximation. The effect will be an increases of our estimation for $\Delta=E_{p}-E_{d}$ by $U_{p} \frac{\bar{n}_{p}}{2}+U_{p d}\left(\bar{n}_{d}-\bar{n}_{p}\right)$, where $\bar{n}_{d}$ and $\bar{n}_{p}$ are the average occupation of $\mathrm{Cu}$ and respectively $\mathrm{O}$ bands. A choice of $U_{p p}=6 \mathrm{eV}, U_{p d}=$ $1.3 \mathrm{eV}$ and $\bar{n}_{p}=0.3$ results in a increase of $\Delta$ by $1.3 \mathrm{eV}$.

To conclude, we take in Eq. 2] $t_{p d}=1.3 \mathrm{eV}, t_{p p}=$ $0.65 \mathrm{eV}, \Delta=4.8 \mathrm{eV}$ and $U=8.8 \mathrm{eV}$.

\section{B. DCA technique}

The DCA is an extension of the Dynamical Mean Field Theory (DMFT) ${ }^{22}$. The DMFT maps the lattice problem to an impurity embedded self-consistently in a host and therefore neglects spatial correlations. The DCA maps the lattice to a finite-sized cluster embedded in a host. Non-local correlations up to the cluster size are treated 


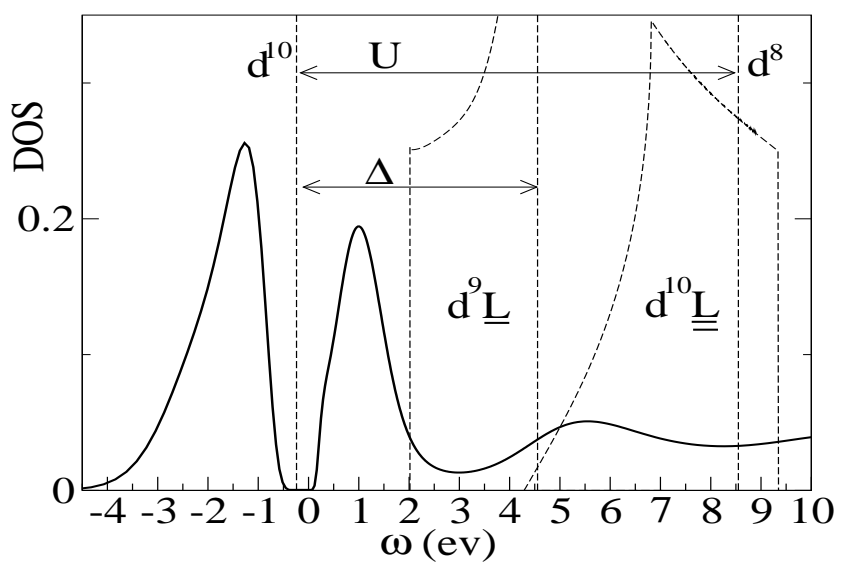

FIG. 2: Two-band Hubbard model DOS at $0 \%$ doping. The solid line is the $d$ part of the DOS calculated at $T=$ $685 \mathrm{~K}$. The value of the parameters is $t_{p d}=1.3 \mathrm{eV}, t_{p p}=$ $0.65 \mathrm{eV}, \Delta=4.8 \mathrm{eV}, U=8.8 \mathrm{eV}$. The dashed line shows the DOS when $t_{p d}=0$. The chemical potential $\mu=0$.

explicitly, while the physics on longer length scales is treated at the mean field level. This technique is ideal for the problems where short-ranged correlations are predominant as in the high- $T_{c}$ materials. Here we calculate the properties of the embedded cluster with a Quantum Monte Carlo (QMC) algorithm. The cluster self-energy is used to calculate the properties of the host, and this procedure is repeated until a self-consistent convergent solution is reached. The self-energy and vertex functions of the cluster are then used to calculate lattice quantities. A detailed description of the QMC-DCA algorithm is given in ref ${ }^{23}$

In the two-band model the oxygen degrees of freedom are not correlated, and therefore they are not included explicitly in the cluster. Their effect is fully contained in the cluster-host hybridization function and in the host Green's function.

Here we consider a $2 \times 2$ cluster of $\mathrm{Cu}$ ions, which we believe to be large enough to capture the essential physics of Hubbard-type models. The $2 \times 2$ cluster will result in a coarse-graining of the BZ in four cells, as shown in Fig. [1]

\section{TWO-BAND HUBBARD MODEL RESULTS}

The undoped materials have one hole per $C u O$ unit. For $t_{p d}=0$ the DOS is given by the dashed line in Fig. 2 and the hole addition states would be of pure $\mathrm{O}$ character. When the $\mathrm{Cu}-\mathrm{O}$ hybridization $t_{p d}$ is switched on, the extra holes added to the oxygen band will scatter with the $C u$ spins and bound states will appear at the bottom of the oxygen band. This is illustrated by the solid line, which plots the partial $d$ DOS which was obtained using the Maximum Entropy Method (MEM) ${ }^{24}$ for the analytic continuation of the QMC data to real frequencies. It can be noticed that the first hole addition states have a strongly mixed $d$ and $p$ character (the $d$

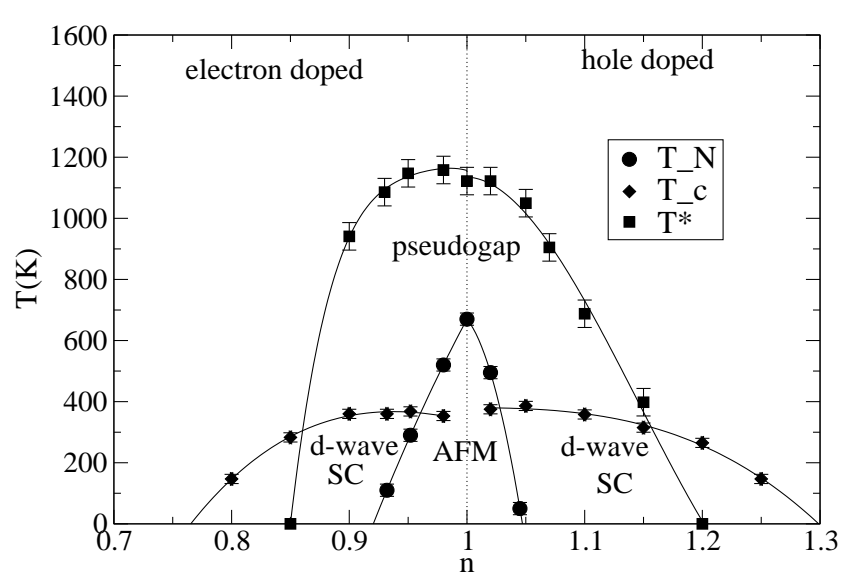

FIG. 3: Two-band Hubbard model phase diagram.

character in the spectrum is large now) and an energy pushed well below the edge of the initial non-bonding oxygen band. Therefore only these states are relevant for the low-energy physics 34 . In the ZR theory these low-energy states which appear as a consequence of the strong $\mathrm{Cu}-\mathrm{O}$ hybridization are considered to be local singlets which move through the lattice like holes in an AFM background. Consequently the claim is that the physics can be described by a one-band t-J model.

In order to determine the phase diagram we calculate a large number of susceptibilities which are relevant for spin, charge and superconducting ordering, both at the center and the corner of the BZ. For example, the Néel and SC critical temperatures, $T_{\mathrm{N}}$ and respectively $T_{c}$ in the phase diagram presented in Fig. 3 are determined from the divergence of the corresponding susceptibilities. The pseudogap crossover temperature $T^{*}$ is obtained from the maximum in the uniform magnetic susceptibility when accompanied by a suppression of spectral weight in the DOS. Similar to what was found in the single-band Hubbard model ${ }^{25}$, we find AFM and d-wave $\mathrm{SC}$ for both electron and hole doped regimes. However the electron-hole symmetry is broken. In the electron doped case AFM persists to a much larger doping. On the contrary, SC disappears at a smaller critical doping 35 . These features of the phase diagram are in qualitative agreement with the experimental findings ${ }^{2}$.

The one-electron spectral functions as measured with photoemission are also different. Our $2 \times 2$ cluster divides the BZ into four cells around $K=(0,0),(0, \pi),(\pi, 0)$ and $(\pi, \pi)$ (see Fig. (11) and approximates the lattice selfenergy by a constant $\Sigma(K, \omega)$ within a cell. Because of this coarse-graining, a comparison with ARPES is not possible apart from gross features. However, as the phase diagram shows, we believe that even our small cluster captures much of the physics of the cuprates. Here we want to stress the difference between the electron and the hole doped case within our $2 \times 2$ cluster approximation. In Fig. 4 a) and b) we show the total $d$ states DOS and the $d$ coarse-grained $K$ dependent DOS (which 


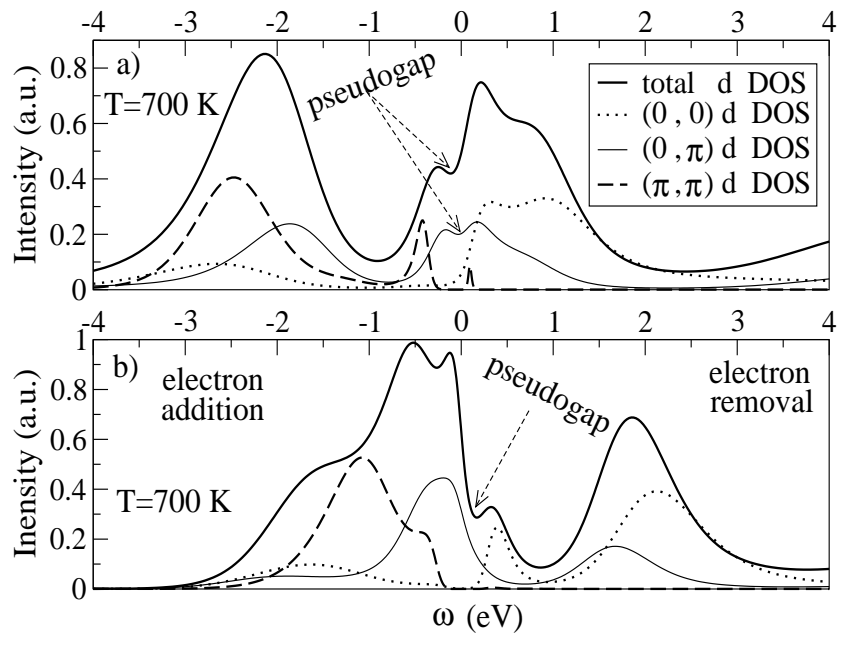

FIG. 4: Total $d$ DOS and coarse-grained $K$ dependent $d$ DOS at $5 \%$ doping. a) hole doping case. b) electron doping case.

would correspond to the average over all $k$ belonging to a coarse-graining cell of the single particle spectra $A(k, \omega)$ ) for the hole and respectively for the electron doped case, at $5 \%$ doping. The total DOS looks qualitatively similar and at the chemical potential we see in both cases a depletion of states which indicates the presence of the pseudogap. The $K$ dependent DOS is very different. The important feature which we want to stress is the location of the pseudogap in the BZ. In the hole doped case the pseudogap appears around $(0, \pi)$. For the electron doped case we do not detect any suppression of states around $(0, \pi)$ even though the pseudogap is clearly present in the total DOS. These features are in agreement with the photoemission experiments. The hole doped materials show Fermi pockets around $(\pi / 2, \pi / 2)$ and gapped states around $(0, \pi)^{3}$. For the electron doped materials the photoemission spectra ${ }^{4}$ exhibit a gap near $(\pi / 2, \pi / 2)$ and Fermi surface pockets around $(0, \pi)$. With the present cluster size the DCA cannot determine where in $k$-space the pseudogap is, but it is interesting that it is not at $(0, \pi)$. The presence of the pseudogap at $(\pi / 2, \pi / 2)$ for the electron doped system can only be checked by increasing the cluster size, and this work is in progress.

The electron and the hole doped susceptibility functions are also different both for the divergence temperatures and the temperature and doping dependence. In Fig. 5 we show the uniform spin and charge susceptibilities versus temperature at $5 \%$ and $10 \%$ doping. A common feature for all cases is the existence of a characteristic temperature $T^{*}$ below which the spin response is suppressed and the charge one is enhanced. $T^{*}$ corresponds to the pseudogap (seen in the DOS) onset temperature. The suppression of the spin excitations below $T^{*}$ was also seen in $\mathrm{NMR}^{26}$ experiments and it was associated with the pseudogap. Besides these common features the electron and the hole doped susceptibilities behave differently. Generally, the maximum value of the spin susceptibility increases with hole filling. This means

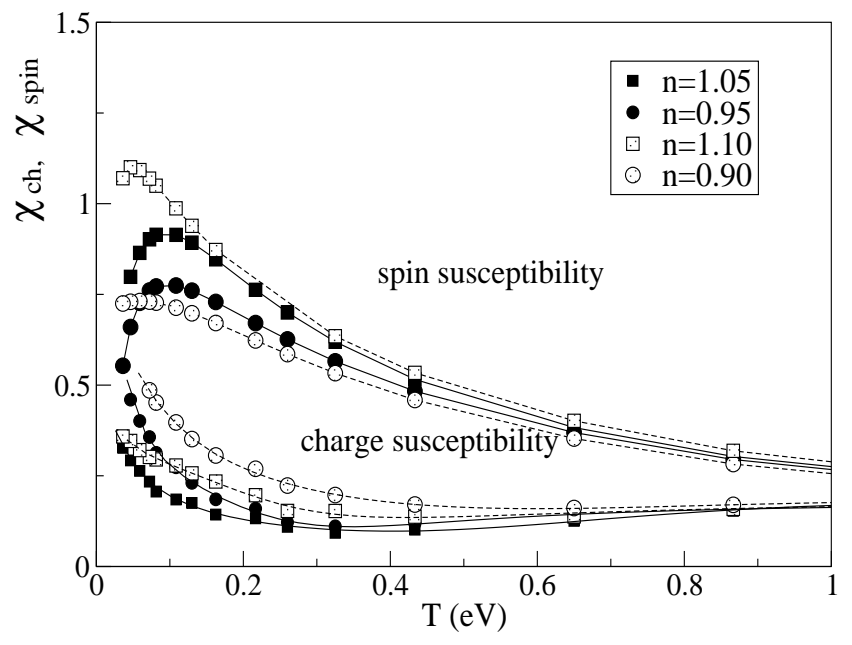

FIG. 5: Uniform spin $\chi_{\text {spin }}$ (upper part) and charge $\chi_{c h}$ (lower part) susceptibilities versus temperature for different hole densities. $n$ in the legend represents the number of holes per unit cell.

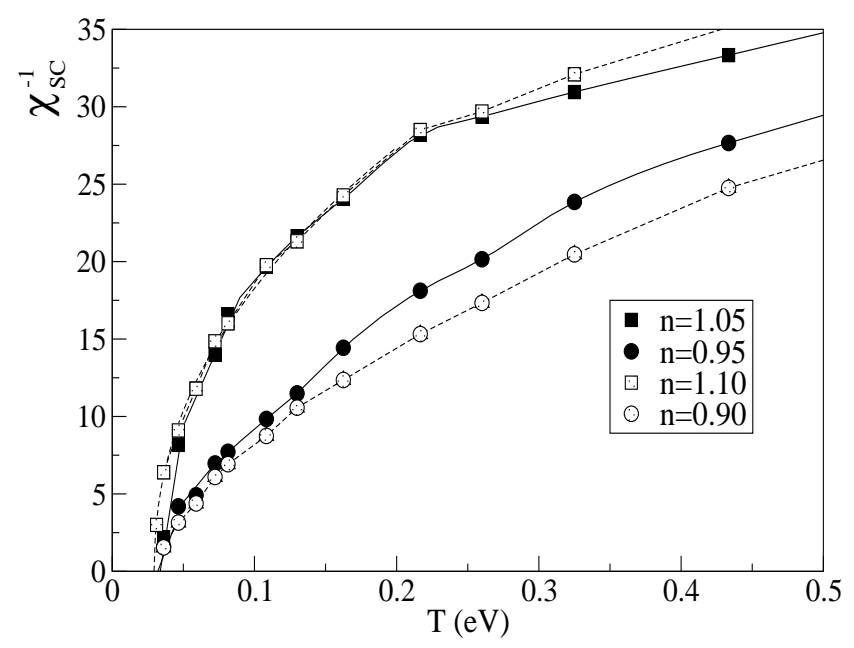

FIG. 6: Inverse of the $d$-wave pairing susceptibility $\chi_{S C}^{-1}$ versus temperature for different hole densities.

that in the hole doped case, the spin susceptibility at the pseudogap temperature is strongly increasing with doping unlike in the electron doped case where it decreases upon doping. At the same doping the hole doped spin susceptibility is much larger than the electron doped one. Another interesting feature is the very strong increase of the charge susceptibility for the electron doped case in the underdoped region (5\% doping), suggesting a tendency towards phase separation 27 .

Asymmetric behavior can also be noticed in Fig. 6] where we plot the inverse of the $d$-wave paring susceptibility. Above $T_{c}$ the pairing susceptibility increases with doping in the electron doped case and remains more or less constant in the hole doped case.

Because of the large $\mathrm{Cu}-\mathrm{O}$ hybridization the system is strongly covalent. For example in the undoped regime 


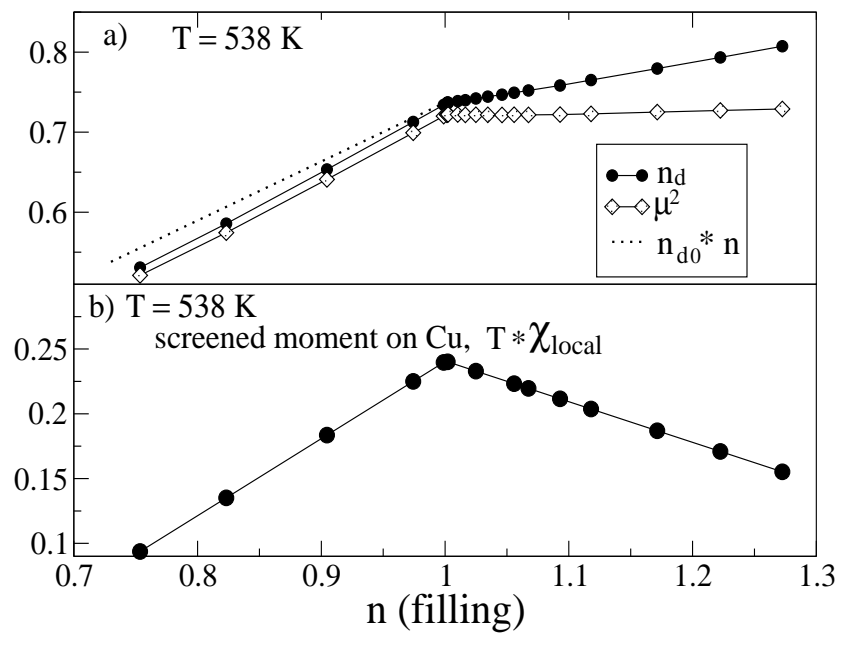

FIG. 7: a) The $\mathrm{Cu}$ occupation number, $n_{d}$, the unscreened $\mathrm{Cu}$ moment, $\mu^{2}$ (Eq. 5) versus hole filling. b) The screened $\mathrm{Cu}$ moment, $T$ 站al (Eq. 6) versus hole filling.

the $\mathrm{Cu}$ occupation number is only $\approx 73 \%$. The fact that the cuprates are strongly covalent was also observed in NMR measurements 28 . We notice that the system exhibits a slightly doping dependent covalency. This is shown in Fig. (7) a where the $\mathrm{Cu}$ occupation number versus hole density is plotted. A constant covalency, equal to the one in the undoped regime (i.e. $0.73 \mathrm{Cu}$ holes and 0.27 O holes per site), would correspond to the dashed line. It can be noticed that, for the electron doped regime, the $\mathrm{Cu}$ hole occupation number is decreasing faster than the hole concentration, which indicates an increasing covalency with increasing electron doping. This happens because at large electron doping, i.e. when the hole-filling of the $\mathrm{CuO}_{2}$ plane is small, the effective hybridization is a result of a large $V(k)$ in the $\mathrm{BZ} 36$. Increasing the number of holes, the BZ starts to fill up and a smaller $V(k)$ will be responsible for the hybridization, and consequently the covalency decreases. For the hole doped regime, the extra holes go primarily on the oxygen band, and therefore we do not have a direct measure of the covalency.

In Fig. [7 the unscreened moment on the $\mathrm{Cu}$ orbitals is shown. It is defined as:

$$
\mu^{2}=\left\langle\left(n_{d i \uparrow}-n_{d i \downarrow}\right)^{2}\right\rangle=n_{d}-2\left\langle n_{d i \uparrow} n_{d i \downarrow}\right\rangle .
$$

The difference between $n_{d}$ and $\mu^{2}$ is a measure of the double occupancy with holes on $\mathrm{Cu}$ sites. In the electron doped regime the double occupancy is very small, but it increases substantially in the hole doped regime, which indicates that the low-energy hole addition states contain double occupied $\mathrm{Cu}$ configurations in a significant measure.

In Fig. 7 7.b the screened moment on $\mathrm{Cu}$, defined as

$$
T \chi_{\text {local }}=\frac{T}{N} \sum_{i} \int_{0}^{\beta}\left\langle S_{i}^{-}(\tau) S_{i}^{+}(0)\right\rangle d \tau,
$$

where $S_{i}$ is $\mathrm{Cu}$ spin operator at site $i$, is shown. The main effect of the extra holes is to screen the spins on the $\mathrm{Cu}$ sites. The screening starts to be effective bellow temperatures of about $\approx 0.5 \mathrm{eV}$ (not shown). In the Zhang-Rice scenario an extra hole perfectly screens one spin on $\mathrm{Cu}$ forming a strongly bound on-site singlet which would contain a significant amount of the double occupied $\mathrm{Cu}$ configuration. So, our results do not contradict the ZR theory, but also do not exclude other scenarios where the extra holes form more complicated bound states which involve more than one $\mathrm{Cu}$ spin. Quantitative analysis based on the amount of screening as function of hole doping cannot give an answer to the validity of the ZR assumption, because, aside from the screening due to the oxygen holes, there are also non-local processes which contribute to the screening of $\mathrm{Cu}$ moments (for example, a possibility is the formation of inter-site spin singlets associated with the Resonance Valence Bond scenario).

\section{REDUCTION TO SINGLE-BAND HUBBARD MODEL}

Concluding that the electron-hole asymmetry is an intrinsic property of the $\mathrm{CuO}_{2}$ plane, we next address the cause of this asymmetry and the possible reduction to a one-band model.

In Sec. III we showed that, due to the $\mathrm{Cu}-\mathrm{O}$ hybridization, the addition of holes results in the formation of low-energy states, with an energy well bellow $(\approx 1 \mathrm{eV})$ the initial oxygen band (see Fig. 2). The reduction to a one-band model is based on the ZR claim that these states are singlets, i.e. spinless entities which can be regarded as holes moving in an antiferromagnetic background. Due to the Monte Carlo nature of our calculation, which does not provide a wave function for the ground state, we cannot determine directly the exact nature of these states. The most we can do is to compare the results of a two-band Hubbard model calculation with those of a one-band Hubbard model, and based on the similarities and differences that we might find to decide about the validity of the single-band approach.

\section{A. Zhang \& Rice approximation and the derivation of the effective parameters}

In order to compare the two-band and the one-band models, we should first get an idea about the possible single-band model effective parameters. We discuss here two different approaches for calculating these parameters, both based on the assumption that the low-energy states are localized, and close to the ZR proposed singlets. 
\begin{tabular}{|l|l|l|l|l|l|l|l|l|l|l|}
\hline cell-perturbation & $U=3.04$ & $J=0.25$ & $J^{\prime} \approx 0$ & $t^{h}=0.477$ & $t^{e}=-0.35$ & $t^{J}=0.433$ & $t^{h^{\prime}}=-0.03$ & $t^{e \prime}=-0.016$ & $t^{J^{\prime}}=-0.003$ \\
\hline cluster calcus & & $J=0.192$ & $J^{\prime}$ & 0.012 & $t^{h}=0.452$ & $t^{e}=-0.323$ & &
\end{tabular}

TABLE I: First row: parameters in eV calculated using cell-perturbation theory. Second row: parameters in eV calculated using cluster diagonalization.

\section{Cell-perturbation theory}

The cell-perturbation theory ${ }^{18}$ assumes that the ZR mapping is strictly true and therefore the low-energy states are genuine local singlets. Here and everywhere in the paper by local we refer to the oxygen orthogonal Wannier states which are different from the non-orthogonal plaquette states around the $\mathrm{Cu}$ ions.

To deduce the one-band model parameters we work in the site representation. We can Fourier transform Eq. 2 and write it as

$$
H=H_{0}+H_{1}
$$

where

$$
\begin{aligned}
H_{0}=\sum_{i} H_{0 i}= & \sum_{i} \sum_{\sigma}\left(E_{0} c_{i \sigma}^{\dagger} c_{i \sigma}+E_{d} d_{i \sigma}^{\dagger} d_{i \sigma}\right. \\
+ & \left.V_{0}\left(c_{i \sigma}^{\dagger} d_{i \sigma}+h . c\right)\right)+U n_{d i \uparrow} n_{d i \downarrow} .
\end{aligned}
$$

Here $i$ represents the site index. The oxygen operators $c_{i}$ describe the orthogonal Wannier states. The ZR assumption implies that $H_{0}$ is responsible for the formation of the low-energy states (local singlets), and $H_{1}$ will determine the hopping parameters. Therefore the cellperturbation theory provides a means to determine the one-band parameters provided that the $\mathrm{ZR}$ assumption is correct. Elaborate calculations along this line were done in 18 for a variety of multi-band parameters. In a first order approximation in $H_{1}$, the effective $\mathrm{U}$ is given by

$$
U_{e f f}=E^{2}+E^{0}-2 E^{1}
$$

where $E^{2}, E^{1}$ and $E^{0}$ represent the energies of the two (i.e. the $\mathrm{ZR}$ singlet), one (i.e. the bonding state) and respectively zero hole states of Eq. 8 An important point is that $H_{1}$ introduce three types of hoppings. If we denote with $\left|2_{i}>,\right| 1_{i}>$ and $\mid 0_{i}>$ the lowest energy states of $H_{0 i}$ corresponding to two, one and respectively zero holes, we have the following hopping integrals

$$
\begin{aligned}
& t_{i j}^{h}=<2_{i}, 1_{j}\left|H_{1}\right| 1_{i}, 2_{j}> \\
& t_{i j}^{e}=<0_{i}, 1_{j}\left|H_{1}\right| 1_{i}, 0_{j}> \\
& t_{i j}^{J}=<1_{i}, 1_{j}\left|H_{1}\right| 0_{i}, 2_{j}>
\end{aligned}
$$

$t^{h}$ (Eq. 10) describes the hopping of the ZR singlet, $t^{e}$ (Eq. 11) the hopping of the electron, and $t^{J}$ produces the exchange interaction

$$
J=4 t^{J^{2}} / U_{e f f}
$$

The cell-perturbation theory applied to our model gives the parameters shown in the first row of Table. [1]

We want to point out two things. First, the reduced Hamiltonian in the cell-perturbation theory is a t-t'-J model,

$$
H=-t \sum_{<i, j>} \hat{b}_{i}^{\dagger} \hat{b}_{j}-t^{\prime} \sum_{<<i, j>>} \hat{b}_{i}^{\dagger} \hat{b}_{j}+J \sum_{<i, j>} S_{i} S_{j}
$$

with different hopping parameters for the electron and the hole doped regions and with a value of the exchange interaction not determined by the quasiparticle's hopping $\left(t^{h}\right.$ or $\left.t^{e}\right)$, but, as it is shown in Eq. 13 by $t^{J}$. Therefore, a comparison with a one-band Hubbard model, should be done cautiously. Second, we want to stress that the value of the next-nearest-neighbor hopping terms $\left(t_{e}^{\prime}\right.$ and $\left.t_{h}^{\prime}\right)$ is very small compared to the nearest-neighbor terms. The reason is that the initial oxygen-oxygen hybridization, $t_{p p}$, results in an effective hopping term comparable in magnitude with the one resulting from the copperoxygen hybridization, but with a different sign. This was also remarked in ${ }^{18}$, and turns out to be an important observation for our final conclusions.

\section{Cluster calculation}

The other approach used for determining the parameters of the one-band model is based on a cluster calculation. In order to estimate the nearest-neighbor hopping, the next-nearest-neighbor hopping and the exchange terms, Eskes et al ${ }^{19}$ considered two clusters, $\mathrm{CuO}_{7}$ (which contains two nearest-neighbor $\mathrm{Cu}$ ions) and respectively $\mathrm{CuO}_{8}$ (which contains two next-nearestneighbor $\mathrm{Cu}$ ions). The exchange term is determined as the energy difference between the singlet and the triplet state of two holes on a cluster. For three holes on a cluster, the two energetically lowest states can be very well (98\%) approximated with the bonding and anti-bonding states of a plaquette $Z R$ singlet hopping between the two cells. Therefore, the differences between these two levels is two times the ZR singlet hopping, $t^{h}$. In an analogous way, considering only one hole on a cluster, the electron hopping, $t^{e}$, is determined. Using the cluster approach, our two-band model results in the effective parameters shown in the second row of the Table $\llbracket$ 


\section{Comparison of the two approaches}

It can be immediately noticed that the two approaches produce different parameters, especially regarding the value of the next-nearest-neighbor hoppings. In the cluster calculation we obtain significant next-nearestneighbor hoping terms, $\left|t^{e \prime}\right| /\left|t^{e}\right|=0.22$ and $\left|t^{h^{\prime}}\right| /\left|t^{h}\right|=$ 0.37 with different sign for the hole and respectively electron doped case $\left(t^{h^{\prime}}<0, t^{e^{\prime}}>0\right)$.

The reason for the discrepancy between the two approaches is that, unlike the cell-perturbation method which considers local singlets, the cluster approach considers singlets between a $\mathrm{Cu}$ hole and a oxygen state formed on the plaquette around the $\mathrm{Cu}$ ion. Since the oxygen plaquette states are nonorthogonal it is possible to write them as a linear combination of many orthogonal oxygen states at different sites, i.e. the plaquette singlets are nonlocal states (in the orthogonal base). It should be pointed out that at first glance this non-locality seems irrelevant (the overlap of the local oxygen states with the plaquette states 11.17 is about $96 \%$ ), but apparently it turns out to influence the value of the next-nearestneighbor hopping of the reduced Hamiltonian considerably.

As pointed out by Emery and Reiter $\frac{17}{17}$, the plaquette singlets are in fact an admixture of ZR singlets and ZR triplets and this can result in a significant value of the spin-spin correlation on the oxygen sites 37 . Of course, if the ZR singlet-ZR triplet admixture is significant, this will make a rigorous reduction to a one-band model impossible, because now the low-energy states have also a spin component and therefore cannot be mapped into holes (spinless entities).

It is worth pointing out that, in the cluster approach, the large value of the next-nearest-neighbor hopping terms results solely from the finite oxygen dispersion and the lack of hopping between the copper and the nextnearest neighbor oxygen plaquette state. On the other hand, in the cell-perturbation theory a copper - nextnearest neighbor oxygen hopping term is present. It results in an effective next-nearest-neighbor hopping with a sign different than the one produced via oxygen-oxygen hopping.

\section{B. Possible reasons for the reduction to fail}

We believe that a comparison between the two-band Hubbard model and a single-band Hubbard model should be done with extreme caution. We want to stress the possible problems here.

First, the reduction based on the ZR approximation, which results in a single-band t-J (or t-t'-J) model assumes the strong-coupling limit, i.e. a ratio $U_{\text {eff }} / t \gg 8$ (the two-dimensional bandwidth is $W=8 t$ ). The lowenergy density of states of the two-band model shown in Fig. 2 and Fig. 4 indicates a bandwidth of the order of the gap, showing that we are rather at the

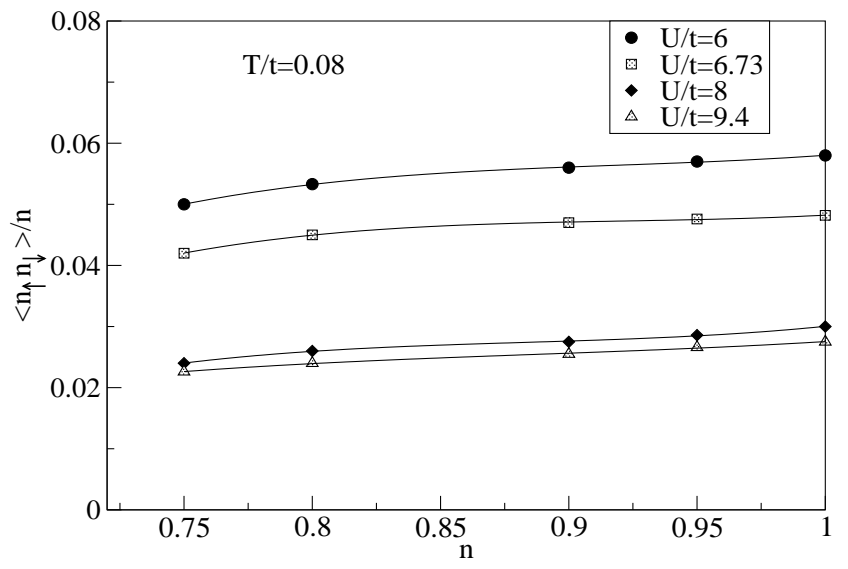

FIG. 8: The relative double occupancy of the orbitals, $\left\langle n_{\uparrow} n_{\downarrow}\right\rangle / n$, versus hole filling, $n$, for different values of the ratio $U / t$ of the single-band Hubbard model.

intermediate-coupling than at strong-coupling. In the cell-perturbation theory we get $U_{e f f} / t^{J}=7.02$, which also suggests intermediate-coupling physics. Therefore, the question to be asked is whether the intermediate coupling regime, characterized by an effective repulsion of the same order of magnitude as the bandwidth, can still be well approximated by a second-order perturbation reduced t-J model.

Second, considering the previous objection, one may think that a reduction to the single-band Hubbard model in intermediate coupling regime, rather than to a t-J model, is more appropriate. However, serious problems arise from the fact that, in the ZR theory the nature of the antiferromagnetic correlations is different from that in the single-band Hubbard model, i.e. it is not directly related with the quasiparticle (ZR singlet or electron) hopping. Therefore, unless both the two-band Hubbard model and the one-band Hubbard model can be reduced to a t-J model, a comparison between them does not make much sense. Nevertheless, we believe that even when the effective repulsion is comparable with the bandwidth the second order perturbation theory which produce the t-J model can be used successfully. We are going to discuss this at the beginning of the next section (Sec. IVC).

Third, the non-locality of the low-energy states (in the sense discussed in Sec. IVA3 can have very serious consequences beyond determining the value of the hopping parameters, making the single-band approach to fail completely.

\section{C. t-t'-U Hubbard model results}

The t-J model results as a low-energy effective Hamiltonian from the Hubbard model by projecting out the doubly occupied states. Therefore, the double occupancy of the site orbitals constitutes a measure of the validity 
of this approximation. In Fig. 8 we plot the double occupancy of the site orbitals for different values of the ratio $U / t$. It can be noticed that for $U / t \geq 6$ the double occupancy is always smaller than $6 \% \frac{38}{2}$. This indicates that, even in the intermediate coupling regime the low-energy physics of the one-band Hubbard model can be well described by a t-J model.

Even if, it is more natural to compare the two-band model with a t-t'-J (or a t-t'-J-J') model, this turns out from our perspective to be rather inconvenient due to the technical difficulties encountered by the QMC when applied to such models. Therefore, we proceed by comparing the two-band model with a t-t'-U Hubbard model, focusing on the qualitative features rather than on a quantitative comparison. In the strong-coupling limit, the t-t'-U model reduces to a t-t'-J-J' model, with the constraint $J^{\prime}=J \times\left(t^{\prime} / t\right)^{2}$. Therefore, it is reasonable to assume that if the value of $\left(t^{\prime} / t\right)^{2}$ is not too large and the reduction of the two-band model to a single-band model is valid, the two models should exhibit similar physics.

Assuming that the reduction to a one-band model in the spirit of the ZR approximation is possible, we should expect from Table. 【the hopping parameters to be different in the hole doped and in the electron doped regions. On the other hand the exchange interaction,

$$
J=\frac{4 t^{2}}{U}
$$

should be the same.

Therefore, we study the single-band t-t'-U Hubbard model and address the following questions:

1) How do the system properties depend on the ratio $t / J ?$

2) What is the role of the next-nearest-neighbor hoping t'?

\section{The $t / J$ dependence}

The values of the parameters in Table. 四 show that in general the ratio $|t / J|$ is larger in the hole-doped regime than in the electron-doped case. In order to address the electron-hole asymmetry observed in the two-band model, in this section we study the properties of the single-band Hubbard model as a function of $t / J$, by keeping $J$ (given by Eq.15) constant and varying the hopping $t$. The next-nearest-neighbor hopping $t^{\prime}$ is set to zero.

With respect to antiferromagnetism, with increasing $t$ the Néel temperature at small doping and the critical doping where the antiferromagnetism disappear decrease. For example, at $5 \%$ doping, the antiferromagnetic susceptibility is diverging only for the small value of $t$ shown in Fig. 9 Assuming that the hole doped regime is characterized by a larger value of $t / J$, this feature is in agreement with the two-band model asymmetric behavior (see Fig. (3).

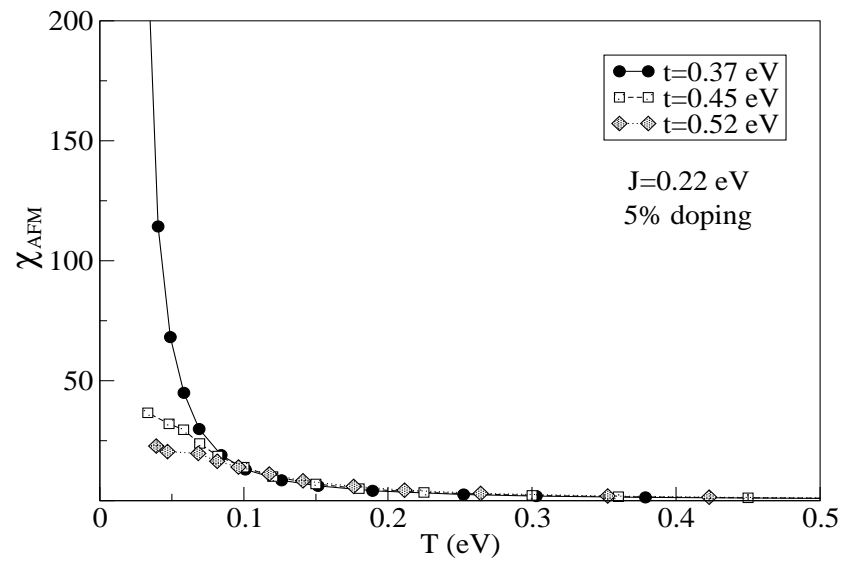

FIG. 9: Antiferromagnetic susceptibility at 5\% doping, for three different values of $t$, when $J$ is constant.

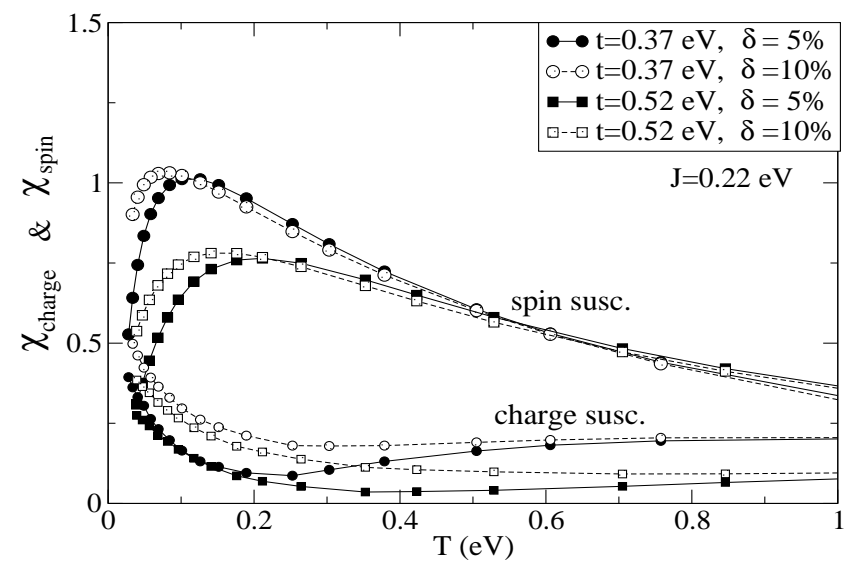

FIG. 10: Spin and charge susceptibilities at 5\% (black) and $10 \%$ (grey) doping for $t=0.37 \mathrm{eV}$ (circle) and $t=0.52 \mathrm{eV}$ (square).

The uniform spin susceptibility is shown in Fig. [10] One can notice that an increase of $t$ results in an increase of $T^{*}$ and a decrease of the spin susceptibility at $T^{*}$. This together with the behavior of the susceptibility as a function of doping is in contrast to what was observed in the two-band model (see Fig. (5) where the spin susceptibility is larger in the hole doped case and an increase (decrease) with doping of the susceptibility at $T^{*}$ for the hole (electron) doped regimes is found.

The behavior of the $d$-wave pairing susceptibility as a function of $t$ is shown in Fig. 11] The critical temperature increases with increasing $t$ (the increase of $T_{c}$ is about $10 \%$ of the increase of $t$ ), as can be seen in the inset (a). This increase is much too large to be in agreement with the two-band model results even if, actually, for the two-band model we obtained a hole-doped $T_{c}$ larger with about $20 \mathrm{~K}$ than the electron-doped one ${ }^{39}$. By extrapolating the inverse of the $d$-wave pairing susceptibility at $28 \%$ doping (see inset (b)) it can be concluded that an increase of $t$ results in an increase of the critical doping where SC disappears. We also notice that, at small 


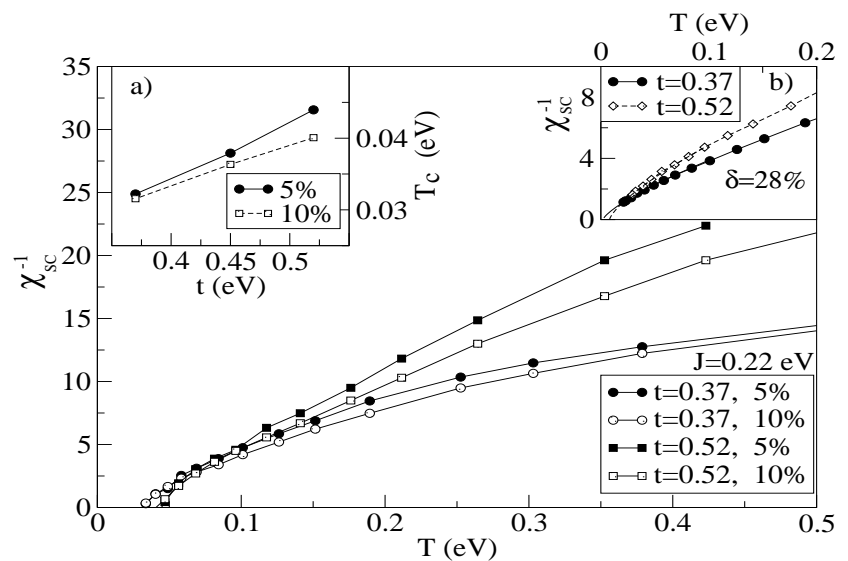

FIG. 11: Inverse of d-wave pairing susceptibility versus temperature for different hole densities and hopping parameters. Inset -a) The critical temperature versus $t$ at $5 \%$ (circle) and $10 \%$ (squares) doping. Inset -b) Inverse of d-wave pairing susceptibility versus temperature at $28 \%$ doping, for $t=0.37 \mathrm{eV}$ (circles) and $t=0.52 \mathrm{eV}$ (diamonds).

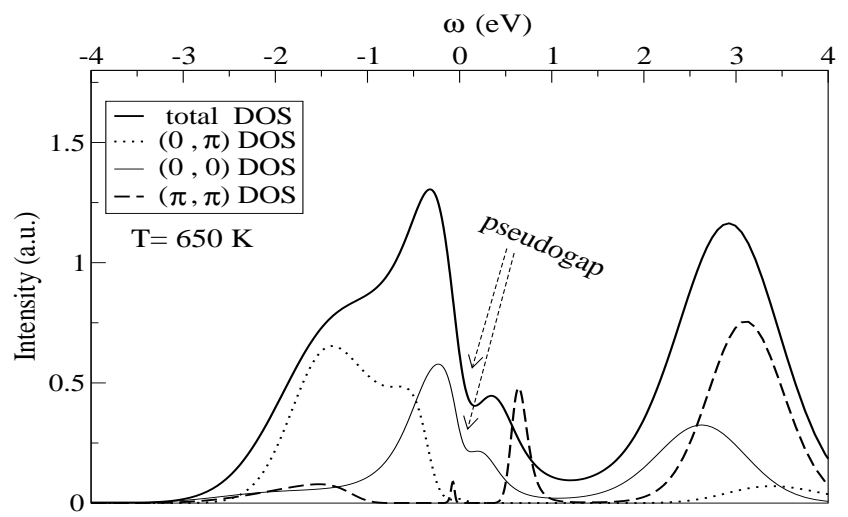

FIG. 12: Single-band t-U Hubbard model total and $K$ dependent DOS at $5 \%$ doping. $J=0.22 \mathrm{eV}, t=0.45 \mathrm{eV}$

doping and above $T_{c}$, a large $t$ suppresses the pairing correlations. These features are in agreement with the asymmetry of the two-band model phase diagram. Nevertheless, we notice that, above $T_{c}$ and for both values of $t$, with increasing the doping the pairing correlations increase too. This behavior is characteristic in the electron doped regime of the two-band model, but cannot explain the hole doped regime where the pairing does not depend on the doping (see Fig. 6). The other difference between the two-band and the single-band Hubbard model is the value of the SC susceptibility critical exponent $\gamma$, which is much smaller in the two-band model case.

The density of states and the $K$ dependent DOS for the one-band t-U Hubbard model at $5 \%$ doping is shown in Fig. 12 The one-particle spectra exhibit a pseudogap in the total DOS and in the $K$ dependent DOS at $(0, \pi)$ point in BZ, similar to the hole doped spectra of the two-band Hubbard model. The single-band t$\mathrm{U}$ Hubbard Hamiltonian is particle-hole symmetric and

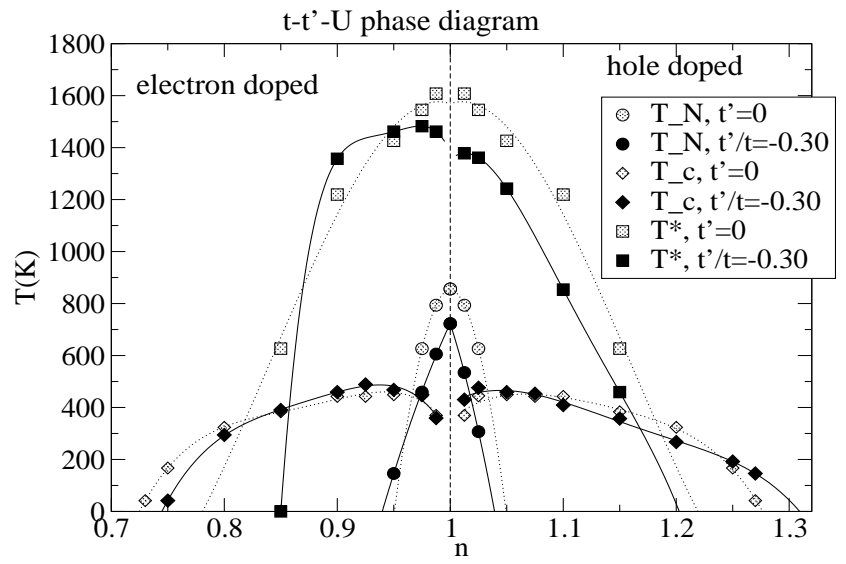

FIG. 13: t-t'-U Hubbard model (solid line) and t-U Hubbard model (dashed line) phase diagrams for $t=-0.45 \mathrm{eV}, U=$ $3.6 \mathrm{eV}$. For the t-t'-U Hubbard model $t^{\prime} / t=-0.3$.

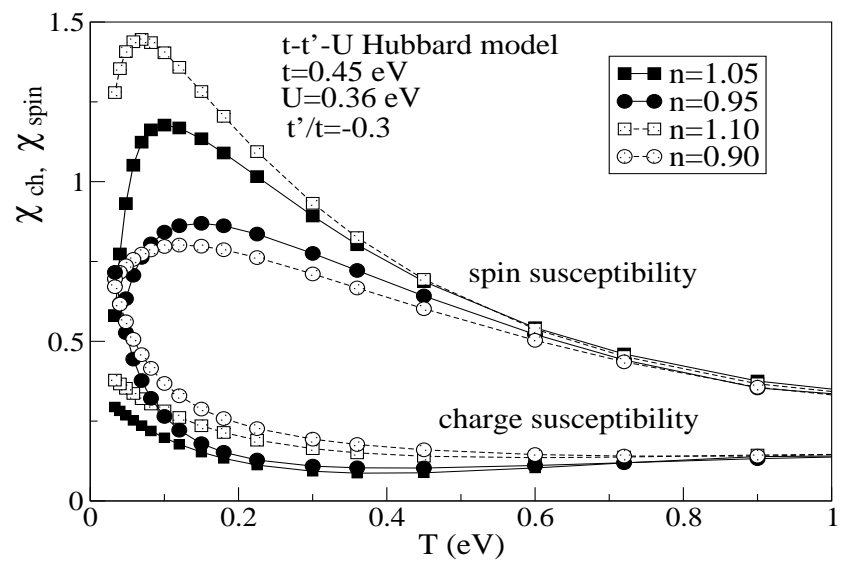

FIG. 14: t-t'-U Hubbard model. Uniform spin $\chi_{\text {spin }}$ (upper part) and charge $\chi_{c h}$ (lower part) susceptibilities versus temperature for different hole densities.

therefore cannot explain the one-particle spectra in the electron-doped regime of the two-band Hubbard model.

At the end of this section we conclude the following: A single-band t-U Hubbard model (i.e. $t^{\prime}=0$ ) with a larger value of the hopping parameter for the hole doped regime cannot explain the electron-hole asymmetries observed in the two-band Hubbard model, especially the ones which characterize the one-particle spectral functions and the susceptibility functions.

\section{The t' dependence}

In this section we study the role of the next-nearestneighbor hopping, $t^{\prime}$, in the single-band Hubbard model

$$
H=-t \sum_{<i, j>} b_{i}^{\dagger} b_{j}-t^{\prime} \sum_{<<i, j>>} b_{i}^{\dagger} b_{j}+U \sum_{i} n_{i \uparrow} n_{i \downarrow} .
$$

We choose the following parameters, $U=3.6 \mathrm{eV}, t=$ $-0.45 \mathrm{eV}$ and $t^{\prime}=0.15 \mathrm{eV}$. These parameters are close 


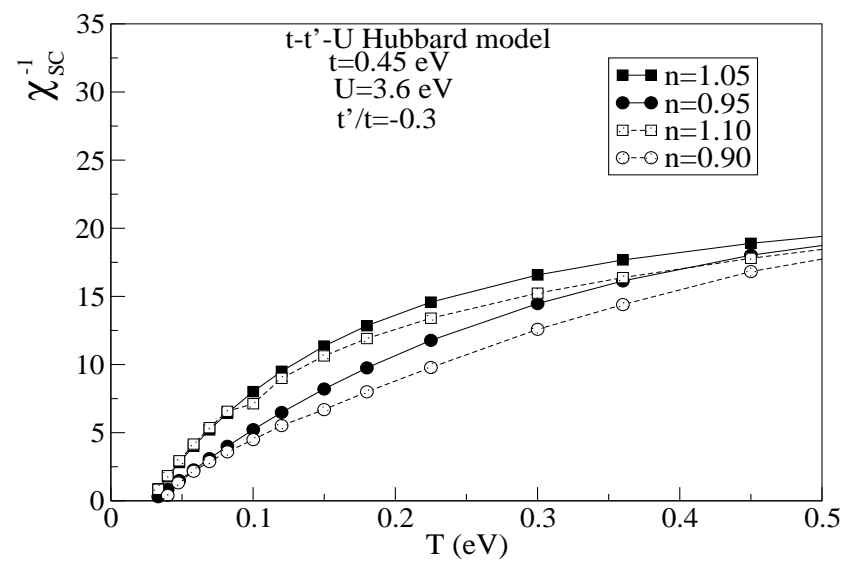

FIG. 15: t-t'-U Hubbard model. Inverse of the $d$-wave pairing susceptibility $\chi_{S C}^{-1}$ versus temperature for different hole densities.
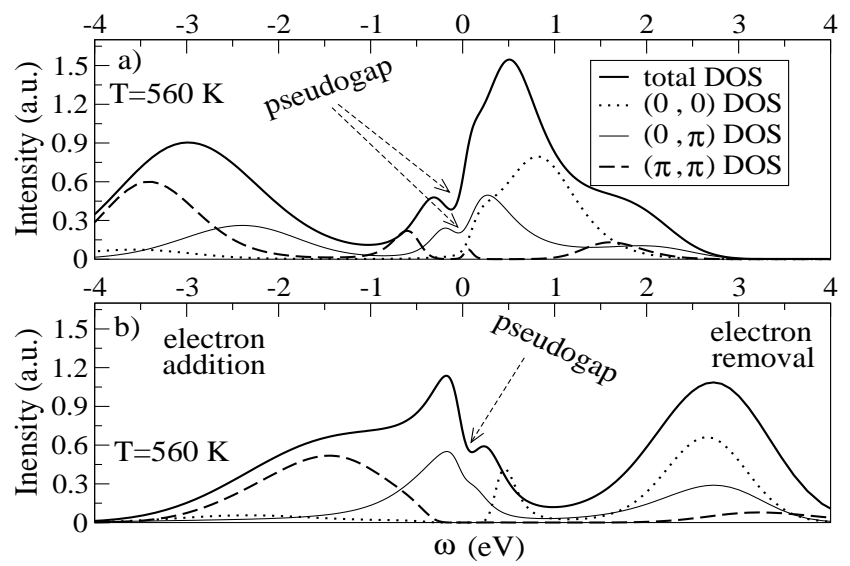

FIG. 16: a) $t-t^{\prime}-U$ total DOS and coarse-grained $K$ dependent DOS at $5 \%$ doping for $t=-0.45 \mathrm{eV}, t^{\prime} / t=-0.3, U=$ $3.6 \mathrm{eV}$. a) hole doping case. b) electron doping case.

to the ones in Table. \ resulting in $J=0.22 \mathrm{eV}$ and $J^{\prime}=0.02 \mathrm{eV}$.

As for the two-band Hubbard model, we work in the hole representation, defined as the one where the filling $1+\delta$ corresponds to a hole doping $\delta$. Values of the filling smaller than one correspond to the electron doped regime. We keep the sign of $t^{\prime}$ always positive. In order to avoid confusion we want to point out that in a t-J model the filling is always smaller than one. Therefore, in order to describe the electron and the hole doped regimes one has to employ the hole and, respectively, the electron representation. Accordingly, the sign of $t^{\prime}$ has to be chosen negative in the hole doped regime and positive in the electron doped case $e^{40}$.

In Fig. 13 the phase diagram of the t-t'-U model is shown with a solid line. For comparison, the phase diagram of $\mathrm{t}-\mathrm{U}$ Hubbard model (i.e. $t^{\prime}=0$ case), which is symmetric with respect to hole and electron doping, is shown with dashed line. At half filling $t^{\prime}$ introduces an effective antiferromagnetic exchange $J^{\prime}=4 t^{2} / U$ be- tween the same sublattice spins and subsequently frustrates the lattice. However at finite electron doping, $t^{\prime}$ favors the antiferromagnetism, making it persist up to a larger doping. On the other hand, in the hole doped case, the antiferromagnetism is always suppressed by $t^{\prime}$. With respect to superconductivity, the presence of $t^{\prime}$ results in a smaller (larger) critical electron (hole) doping at which the superconductivity disappears. The asymmetry introduced by $t^{\prime}$ is in agreement with the one observed in the two-band model phase diagram. We find that $t^{\prime}$ has no major influence on the maximum superconductivity critical temperature $T_{c}^{\max }$.

The uniform spin and charge susceptibilities are shown in Fig. 14 The spin susceptibility at the pseudogap temperature $T^{*}$ is strongly increasing with doping for the hole doped case and an opposite effect is seen for the electron doped case. The downturn at $T^{*}$ in the spin susceptibility is much sharper for the hole doped regime indicating a fast transition to the pseudogap physics. All these features are in very good qualitative agreement with the ones corresponding to the two-band Hubbard model. Because of the similarity with the two-band model, it is also worth mentioning that in the electron-doped regime the charge susceptibility is strongly increased below $T^{*}$ in the underdoped region.

The $d$-wave paring susceptibilities shown in Fig. 15] exhibit asymmetric features, also in a qualitative agreement with those in the two-band model. In the electron doped regime, with increasing the doping, the pairing correlations above $T_{c}$ increase. In the hole-doped regime close to $T_{c}$, the pairing correlations do not significantly depend on the doping. However, contrary to the two-band model behavior, at larger temperature an increase of pairing correlations with doping is observed. The magnitude of this increase is smaller than in the electron-doped case and a larger value of $t^{\prime}$ (e.g. $t^{\prime} \approx 0.4 t$, not shown) will reduce it further, improving the resemblance with the two-band model.

In Fig. [16] we present the DOS of the t-t'-U Hubbard model at $5 \%$ doping. The one-particle spectral functions resemble the corresponding two-band Hubbard model ones. The presence of the $t^{\prime}$ parameter is responsible for the location of the pseudogap in the BZ.

The necessity of the $t^{\prime}$ in explaining the measured ARPES line shape and the electron-hole asymmetry was realized early on ${ }^{31.32}$. Representing hoppings in the same sublattice, this parameter is not severely renormalized by the AFM background, and consequently its influence turns out to be important. Exact diagonalization results ${ }^{32}$ of a t-t'-J model are in agreement with ours. The $t^{\prime}$ hopping process lowers the kinetic energy and moves the quasiparticle position from $(\pi / 2, \pi / 2)$ to $(0, \pi)$ in the electron doped case . The Néel-like configurations, which do not hinder this process, are stabilized. In the hole doped case the $t^{\prime}$ hopping does not lower the kinetic energy of quasiparticles and it is not energetically favorable, therefore leading to a suppression of AFM at all dopings.

The main conclusion of this section is that a one-band 
t-t'-U Hubbard model describes qualitatively well the physics (i.e. the phase diagram, the one-particle spectra and the two-particle response functions) of the two-band Hubbard model, provided a significant value of the nextnearest neighbor hopping $\left(t^{\prime} / t \approx 0.2-0.5\right)$ is considered. However, besides all these similarities there are also some important differences which we are going to emphasize in the next section.

\section{DISCUSSION}

In general, the deduction of an effective low-energy Hamiltonian implies two steps. First, defining the lowenergy states and second, projecting the resolvent operator, $G(E)=(E-H)^{-1}$, on the subspace spanned by these low-energy states ${ }^{33}$. The inverse of the projected operator can be written as $E-H_{\text {eff }}(E)$, where $H_{\text {eff }}$ is the low-energy Hamiltonian ${ }^{41}$. This procedure is equivalent to finding an Hamiltonian which produces the same one-particle, two-particle, three-particle, etc., spectral functions on the energy range considered to be "low-energy".

Rigorously, in order to prove that the one-band model is the effective Hamiltonian which describes the two-band Hubbard model low-energy physics we should compare not only the one-particle and the two-particle spectra, but also all higher order correlation functions. However, we believe that the comparison of only the oneparticle and the two-particle spectral functions is compelling enough, especially since the experimental information is also obtained by measuring the response functions behavior (and in almost all cases the two-particle operators or the one-particle ones, as in photoemission, are involved). It is also true that a comparison of the dynamic susceptibilities would be required, but with our Quantum Monte Carlo based algorithm the calculation of these quantities for the two-band model is extremely computational resources consuming and has not been done yet. However partial information about the relevant excited states is contained in the temperature behavior of the static susceptibilities.

The main conclusion of Sec. IV] is that a t-t'-U Hubbard model describes qualitatively well the physics of the two-band Hubbard model, but only if a substantial next-nearest-neighbor hopping is considered. However, the calculation in Sec. IV A 1 (first row of Table. II) and the more rigorous results by Feiner et al. ${ }^{18}$, show that in a strict ZR picture the next-nearest-neighbor hopping is negligible. Therefore it is difficult to explain the twoband Hubbard model physics assuming the formation of local ZR singlets. For hole doped systems, a significant value of $t^{\prime}$ can be obtained only if the extra holes form nonlocal bound states with the existing $\mathrm{Cu}$ holes, presumably something close to the plaquette singlets. Of course we have no reasons to discard other states spread over even more oxygen sites, which can result in a magnitude of the hopping parameters different (probably not too much) form the one obtained by cluster calculation (second row of Table. I). In the electron doped systems, the doping dependent covalency shown in Fig. 17. a, clearly indicates that the hybridization of the $\mathrm{Cu}$ with the $\mathrm{O}$ states at different sites is important. A doping dependent covalency should also imply doping dependent parameters.

The cluster calculation which allows the formation of non-local (plaquette) low-energy states, unlike the cellperturbation (or strict ZR) approach, provides a value of the hopping parameters which captures qualitatively the physics of the two-band model. However, we do not believe that finding the exact value of the one-band Hubbard model parameters is a relevant or even a well addressed problem, because the non-locality of the lowenergy states implies that the two models are not equivalent, as was pointed out by Emery et al ${ }^{17}$. Aside from the similarities between the two-band and the t-t'-U Hubbard models discussed in SecIVC2 we also find some differences.

For example, one important difference can be observed in the $d$-wave pairing susceptibility (Fig. [15 and Fig. 6) . In the two-band Hubbard model the critical exponent $\gamma$ which defines the divergence of the susceptibility at $T_{c}$, is much smaller (around $\approx 0.4$ at finite hole doping) than the one characteristic to the one-band model (around $\approx$ $0.6)$, indicating larger fluctuations 42 .

Both the cell-perturbation and cluster calculation provide a larger nearest-neighbor hopping $t$ for the hole doped region. According to the analysis presented in Sec. IVC 1 this should result in both larger $T^{*}$ and $T_{c}$. However the two-band model results does not indicate that this is the case, the respective critical temperatures being not very different in the electron and hole doped regimes.

Based on our comparison we can draw the following conclusions. The one-band Hubbard model retains much of the two-band Hubbard model physics, but a significant next-nearest-neighbor hopping $\left(t^{\prime} / t \approx 0.3\right)$ should be provided. If the purpose of the investigation is the study of the basic physics like the SC mechanism, the proximity of AFM, SC and pseudogap we believe that a one-band t-t'-U Hubbard model should be good enough. On the other hand, if the purpose is to describe more subtle features like the ones which may result from the finite value of the spin correlation on oxygen, or if a quantitative material specific calculation is desired, the singleband model approach fails. Obviously also the singleband model should not be used to describe spectral features at energies above $0.5 \mathrm{eV}$ such as the optical, electron energy loss and inelastic x-ray scattering results.

\section{SUMMARY AND CONCLUSIONS}

In this paper we use the DCA to calculate the properties of the two-band Hubbard model. The $2 \times 2$-site cluster phase diagram resembles the generic phase dia- 
gram of the cuprates and exhibits electron-hole asymmetry. We also find asymmetric features for the one-particle spectral functions and for the relevant susceptibility functions. These characteristics are in qualitative agreement with experimental findings.

We address the validity of the single-band Hamiltonian as the effective low-energy model for the cuprates. We discuss the possible problems which may cause the failure of the reduction from two-band to one-band and also show that, depending on the approximations involved, the value of the one-band Hubbard parameters (especially the next-nearest-neighbor hopping) can be significantly different.

We use DCA to study the role of the different parameters in the single-band t-t'-U Hubbard model and compare the phase diagram, the one-particle and the twoparticle response functions with the corresponding twoband Hubbard model ones. We conclude that the two models exhibit similar low-energy physics provided that a significant next-nearest-neighbor hopping $t^{\prime}$ is considered. The parameter $t^{\prime}$ is also the main culprit for the electron-hole asymmetry of the cuprates.

The large value of $t^{\prime}$ needed for a qualitative agreement between the two models cannot be obtained in a strict ZR picture where the extra holes form local singlets with the existing $\mathrm{Cu}$ holes. Plaquette singlets, which in the oxygen Wannier representation are not local, and presumably other spatially extended states can provide a larger value of $t^{\prime}$. The doping dependent covalency in the electron doped case also indicates that the non-local $\mathrm{Cu}-\mathrm{O}$ hybridization is important. However, the formation of non-local low-energy states also implies that they are not real singlets and consequently cannot be rigorously mapped into holes and therefore the two models are not equivalent.

We also point out some differences between the two models. In the two-band Hubbard model the fluctuations in the $d$-wave pairing channel above $T_{c}$ is much stronger. The deduction of the parameters both in cellperturbation and cluster approach results in a larger nearest-neighbor hopping $t$ for the hole doped regime. However the critical temperatures $T^{*}$ and $T_{c}$ in the twoband Hubbard model are approximatively the same in both regimes, quite different from what should be expected.

The conclusion is that a single-band Hubbard model with a significant value of the next-nearest-neighbor hopping $\left(t^{\prime} / t \approx 0.3\right)$, captures the basic physics of the twoband Hubbard model, including the proximity of antiferromagnetism, superconductivity and pseudogap and explaining the electron-hole asymmetry seen in the phase diagram, one-particle and two-particle spectral functions. However, the single-band Hubbard model is not entirely equivalent to the two-band Hubbard one and we believe that it is not suitable for quantitative material specific studies or for describing more subtle features which may results from the non-locality of the low-energy states. It is also not suitable to describe physics which implies excitations with energy scales larger than $\approx 0.5 \mathrm{eV}$.

\section{Acknowledgments}

We thank F.C. Zhang and Paul Kent for useful discussions. The work was supported by NSF grant DMR0073308, by CMSN grant DOE DE-FG02-04ER46129 and by the Netherlands Foundation for Fundamental Research on Matter (FOM) with financial support from the Netherlands Organization for Scientific Research (NWO) and the Spinoza Prize Program of NWO. The computation was performed at the Pittsburgh Supercomputer Center, the Center for Computational Sciences at the Oak Ridge National Laboratory and the Ohio Supercomputer Center. Part of this research was performed by TM as a Eugene P. Wigner Fellow and staff member at the Oak Ridge National Laboratory, managed by UTBattelle, LLC, for the U.S. Department of Energy under Contract DE-AC05-00OR22725.
1 M. H. Hettler, A. N. Tahvildar-Zadeh, M. Jarrell, T. Pruschke and H.R. Krishnamurthy, Phys. Rev. B 58, R7475 (1998); M. H. Hettler,M. Mukherjee, M. Jarrell and H.R. Krishnamurthy, Phys. Rev. B 61,12739 (2000).

2 C. Almasan and M.B. Maple, Chemistry of HighTemperature Superconductors, 1991 edited by C.M.R. Rao; E. Dagotto, Rev. Mod. Phys 66763 (1994)

3 B.O. Wells et al., Phys. Rev. Lett. 74, 964 (1995)

${ }^{4}$ N.P. Armitage et al., Phys. Rev. Lett. 88, 257001 (2002)

5 J. Zaanen, G.A.Sawatzky and J.W.Allen, Phys.Rev.Lett. 55, 418 (1985)

6 J. D. Jorgensen, H. B. Schuttler, D.G. Hinks, D. W. II Capone, K. Zhang, M. B. Brodsky, and D. J. Scalapino, Phys.Rev.Lett. 58, 1024 (1987)

7 L. F. Mattheiss, Phys.Rev.Lett. 58, 1028 (1987)

8 J. Yu, A. J. Freeman and J. H. Xu, Phys.Rev.Lett. 58,
$1035(1987)$

9 C. M. Varma and S. Schmitt-Rink, Solid State Com. 62, $681(1987)$

10 V.J. Emery, Phys.Rev.Lett. 58, 2794 (1987); V.J. Emery and G. Reiter, Phys. Rev. B 38, 4547 (1988)

11 F.C. Zhang and T.M. Rice, Phys. Rev. B 37, R3759 (1988); Phys. Rev.B 41, 7243 (1990)

12 H. Eskes, L.H.Tjeng and G.A.Sawatzky, Phys.Rev. B 41, 288 (1990)

13 E.B. Stechel and D.R. Jennison, Phys.Rev. B 38, 4632 (1988)

14 M.S. Hybertsen, M. Schluter and N.E. Christensen, Phys.Rev. B 39, 9028 (1989)

15 J. Zaanen and A.M. Oles, Phys. Rev. B 37, 9423 (1988)

16 H. Eskes and G.A. Sawatzky, Phys. Rev. Lett. 61, 1415 (1988) 
17 V.J. Emery and G. Reiter, Phys. Rev. B 38, R11938 (1988); Phys. Rev.B 41, 7247 (1990)

18 J. H. Jefferson, H. Eskes and L. F. Feiner, Phys. Rev.B 45, 7959 (1992); L. F. Feiner, J. H. Jefferson, and R. Raimondi, Phys. Rev.B 53, 8751 (1996)

19 H. Eskes, G.A.Sawatzky and L. Feiner, Physica C 160, 424 (1989)

20 A. K. McMahan, J.F. Annett, R.M. Martin, Phys. Rev. B 42, 6268 (1990)

21 A. Macridin, Ph.D. thesis, University of Groningen, http://www.ub.rug.nl/eldoc/dis/science/a.macridin

22 A. Georges G.Kotliar, W.Krauth and M.Rozenberg, Rev.Mod.Phys. 68, 13 (1996)

23 M. Jarrell, Th.Maier, C.Huscroft, and S.Moukouri, Phys.Rev. B 64,195130 (2001)

${ }^{24}$ M. Jarrell and J. E.Gubernatis, Phys.Rep. 269, 135 (1996)

25 M. Jarrell, Th.Maier, M.H.Hettler, A.N.Tavildarzadeh, Europhys.Lett. 56, 563 (2001)

${ }^{26}$ W.W. Warren et al., Phys. Rev. Lett. 62, 1193 (1989); M. Takigawa et al., Phys.Rev.B 43, 247 (1991); H. Alloul, A. Mahajan, H.Casalta, O. Klein, Phys. Rev. Lett. 70, 1171 (1993)

27 A. Macridin et al., in preparation.

28 M. Takigawa, et al. Physica C 162-164, 853 (1989); M. Takigawa High-Temperature Superconductivity, edited by K.Bedell, D. Coffey, D.E. Meltzer, D. Pines, and J.R. Schriffer (Addison-Wesley, 1990), p. 236

29 S.Moukouri and M.Jarrell, Phys.Rev.Lett. 87, 167010 (2001)

30 Eduardo Fradkin, Field theories of condensed matter systems, (Redwood City, CA, Addison-Wesley, 1991), p. 9-10

31 A. Nazarenko, K.J.E. Vos, S. Haas, E. Dagotto, R.J. Gooding, Phys. Rev. B 51, R8676 (1995); R. Eder, Y. Ohta, G. A. Sawatzky, Phys. Rev. B 55, 3414 (1997); P.W. Leung, B.O. Wells, R.J. Gooding, Phys. Rev. B 56, 6320 (1997); O. P. Sushkov, G. A. Sawatzky, R. Eder, H. Eskes, Phys.
Rev. B 56, 11769 (1997)

32 T. Tohyama and S. Maekawa, Phys. Rev.B 64, 212505 (2001); Supercond. Sci. Technol.13, R17

33 A.Auerbach, Strongly Interacting Electrons and Quantum Magnetism, (New York, Springer-Verlag, 1994), p. 25.

34 A five-band Hubbard model calculation ${ }^{21}$ confirms that, the occupation number of the non-bonding oxygen bands is less than $1 \%$ up to $40 \%$ hole doping.

35 Our preliminary calculations indicate that the electronhole asymmetry in the phase diagram is more pronounced when larger than $2 \times 2$ clusters are considered.

36 The Cu-O hybridization (Eq. 4) is strongly $k$ dependent, its value taking values from $2 \sqrt{2} t_{p d}$ at $(\pi, \pi)$ point to zero at $(0,0)$ point in the BZ.

37 In their example, Emery and Reiter considered a ferromagnetic alignment of $\mathrm{Cu}$ spins and showed that the $\mathrm{ZR}$ singlet -ZR triplet admixture is even at the $(0,0)$ point in the Brillouin Zone.

38 We also find (not shown) that with decreasing $U / t$ beyond this value, the double occupancy increases fast, being about $15 \%$ for $U / t=4$.

39 Nevertheless we should take the necessary precautions saying that this value is of the order of the error bar (see Fig. 31)

40 Under electron-hole representation change both $t$ and $t^{\prime}$ change sign. A change of $t$ sign has no physical consequences aside from a translation with $(\pi, \pi)$ in the BZ corresponding to a canonical transformation which changes the sign of site orbitals on one sublattice. An equivalent statement is that the particle-hole transformation, as defined in ref ${ }^{30}$, change the sign of $t^{\prime}$ but not of $t$.

$41 H_{\text {eff }}$ can be considered independent of $E$ if the high-energy and the low-energy states are well separated.

42 The deviation of $\gamma$ from the mean-field value 1 is a measure of fluctuation. 